\title{
Acknowledgement to Reviewers of Societies in 2018
}

\author{
Societies Editorial Office
}

MDPI, St. Alban-Anlage 66, 4052 Basel, Switzerland

Published: 12 January 2019

Rigorous peer review is the cornerstone of high-quality academic publishing. The editorial team greatly appreciates the reviewers who contributed their knowledge and expertise to the journal's editorial process over the past 12 months. In 2018, a total of 120 papers were published in the journal, with a median time to first decision of 20.5 days and a median time to publication of 57.5 days. The editors would like to express their sincere gratitude to the following reviewers for their cooperation and dedication in 2018:

\begin{tabular}{ll} 
Adam, Dinham & Benedetto, Barabino \\
Adela, Badau & Benjamin, Bowman \\
Ahmad, Ahmad & Betti, Gianni \\
Aisha, Geissinger & Beverley, Clough \\
Ajantha Sisira, Kumara & Brandy L., Simula \\
Aje, Carlbom & Brenda, Cude \\
Alejandro, Padilla-Rivera & Brennan K., Berg \\
Alex, Channon & Brian S, Carter \\
Alexi, Gugushvili & Brian, Kingshott \\
Alexis M., Silver & Brian, Phillips \\
Almudena, Moreno & Bruce, Maddy-Weitzman \\
Amanda, Lucia & Bruno, Costa \\
Amber, Garcia & Bryan, Duckham \\
Amparo Huertas, Bailén & Bryane, Michael \\
Andrea, Felicetti & Buckingham, Susan \\
Angela, Chang & Camilla, Haavisto \\
Anna, Lavizzari & Camilo, Negri \\
Anna, Lawson & Carlos, Gutiérrez-García \\
Anna, Matheson & Carlos, Teixeira \\
Anna, McFarlane & Carolin, Rapp \\
Anna, Vanzan & Caroline, Doyle \\
Anne, Dezetter & Carrie Packwood, Freeman \\
Antonio M., Jaime-Castillo & Catherine, O'Sullivan \\
Arianne, Conty & Celena Schede, Bergdahl \\
Aris, Mousoutzanis & Chandan, Jha \\
Arthur, Blaser & Charles Che, Fonchingong \\
Auður H, Ingólfsdóttir & Charli, Eriksson \\
Badicu, Georgian & Charlotte, Legg \\
Barbara, Fura & Chiara, Pussetti \\
Barry, Fearnley & Chris, Bobel \\
Barry, Godfrey & Christopher St., Vil \\
Bastian, Becker & Christopher W., Mullins \\
Behnam, Behnia & Christopher, Byrne \\
Ben, Kisby & Claire E., Brolan \\
Ben, Miller & Clemens, Lechner \\
\hline
\end{tabular}


Corey Lee, Wrenn

Crisanta-Alina, Mazilescu

Dale, Connally

Dale, Spencer

Dan-Cristian, Dabija

Daniel, Kavish

Daniel, Klasik

Daniel, Krymkowski

Daniel, López-López

Dave, Martin

David, Weakliem

Deborah, Stienstra

Delia, Ferri

Denia, Garcia

Dennis, Keating

Dennis, McGilvray

Derek H., Alderman

Despina P., Dimelli

Dong, Hoang

Douglas, Pratt

Dovile, Budryte

Dulce, Filgueira

Ebba, Sundin

Elena, Andina-Díaz

Elena, Delavega

Elena, Riza

Elias, Naumann

Elli, Heikkilä

Elsa, Pegado

Emily, Rainsford

Emma, Crawford

Erdal, Atukeren

Erica, Smith

Ernest B., McGowen

Eugène, Loos

Eva, Midden

Eva, Neely

Eyob Balcha, Gebremariam

Fabio, Caiani

Federico, Squarcini

Finn, Mackay

Fiona, Cuthill

Flemming Juul, Christiansen

Floyd, Ormsbee

Foteini, Grivokostopoulou

Frank, Achtenhagen

Fred, Mason

Gabriel, González Valero

Gabriela, Topa

Garson, Hunter

Gary, Pollock

George, Jennings
George, Luta

Greg, Newbold

Gregor, Wolbring

Guglielmo, Lomonaco

Guido, Erreygers

H., Wels

Hadia, Mubarak

Harkavy, Ira

Heather, Piper

Heleen J., Janssen

Howard, Williamson

Ian, Fairlie

Iftikhar, Hussain

Igor, Ryabov

Ihsan, Yilmaz

Inmaculada, García-Martínez

Ionel, Bostan

Isha, Sharma

Isidoros, Perikos

Isidro, Maya-Jariego

Iulia Cristina, Muresan

Ivan, Gololobov

Ivis, Garcia

Jacqueline, Low

James, Campbell

James, Sloam

Jan Anne, Annema

Jan D., Reinhardt

Jane, Essex

Janet, Richmond

Jay, Mitra

Jeff, Brown

Jeff, Gibbons

Jenny, Phillimore

Jesús, Granados-Sanchez

Jill, Stavert

Joan, Wesley

John, Bunce

John, Jacobi

John, Wainwright

Jon, Ord

Joost, Van Hoof

Jose Manuel, Diaz-Sarachaga

Juan Miguel, Campanario

Julia, Koltai

Jülide Yıldırım, Öcal

Justin, Brown

Justine, Howe

Kai, Li

Karen, Jacobsen

Karenza, Moore

Kathleen, Moore 
Katrina, McDonald

Kenneth, Reid

Kimmo, Jokinen

Kristen, Hessler

Kuzey, Yilmaz

Kwok, Ng

Lara, Maestripieri

Larkin, Lamarche

Larry, Nackerud

Laura K, Marsh

Laura, Smith-Khan

Laurah E., Klepinger

Lauren, Martin

Lawrence, Hogue

Layana, Navarre-Jackson

Leah M., Omilion-Hodges

Liberto, Carratalá Puertas

Lili-Ann, Wolff

Lindy-Anne, Abawi

Lisa Jean, Moore

Lorenzo, Pedrini

Louisa, Willoughby

Lucy, Hunt

Luis Gabriel, Carmona

Luis Ricardo, Fraga

Lutz, Kaelber

Lyndal, Sleep

Lyusyena, Kirakosyan

Macià, Blázquez-Salom

Maninder, Setia

Manyu, Li

Marc, Bendick

Marcel-Cristian, Voia

Marco, Giugni

Margaret, Souza

María Dolores, Sánchez-Fernández

Maria Francesca, Murru

Maria José, Sá

Maria Magdalena, Bujnowska-Fedak

María, Napal

Maria, Nyholm

Mariagrazia, Fanchi

Marianne Tierney, Fitzgerald

Marianne, Sarkis

Mario De, Benedittis

Mark, Doidge

Mark, Orams

Mark, Shephard

Martin, Biewen

Martin, Hand

Martina, Ardizzi

Maruice, Mangum
Marvin, Walker

Mary, Crock

Marzena, Starnawska

Masooda, Bano

Mattias, Fatke

Maureen A., Eger

Maximilian, Lakitsch

Maxmillian, Martin

Md Saidul, Islam

Mehek, Muftee

Merve, Sarikaya-Şen

Mette L., Baran

Michael A., Long

Michael A., Stefanone

Michael, Carriere

Michael, Cope

Michael, Dunning

Michael, Lynch

Michał, Rozpara

Michela, Franceschelli

Mickie, Swisher

Miguel Jesús, Medina Viruel

Mindy, Fullilove

Minnie, Thomas

Miriam, Zuk

Moha, Ennaji

Mojtaba, Vaismoradi

Moritz, Hess

Muddassar, Sarfraz

Myia, Williams

Naomi, Farber

Natalie, Zervou

Nate, Breznau

Nathalie, Giger

Nathan, Manning

Nicholas, Allen

Nicola De, Ugolotti

Nik, Summers

Nils, Gustafsson

Nora, Repo-Saeed

Ondřej, Dvouletý

Pat, Thane

Patrick Marius, Koga

Paul, Hodge

Paul, Stretesky

Pei-Kuan, Lin

Peter J., Kivisto

Petra, Schneider

Philip, Kretsedemas

Philippa, Velija

Phillip J., Granberry

Piers, Gooding 
Ping-Ann, Addo

Pooja, Sawrikar

Priya, Saha

Quazi Mohd Mahtab-uz, Zaman

Rafael M., Martins

Rafał, Dreżewski

Rahbel, Rahman

Rakhshanda, Khan

Raul Navarro, Olivas

Ray, Block Jr

Rebeca Cristina López, González

Rhona, Winnington

Richard, Waldron

Rifat, Akhter

Rika, Haga

Rob, Watts

Robert J., Pauly

Robert Vann, Rikard

Robert, Dinerstein

Robert, Markley

Robert, Stock

Roberto, Impicciatore

Roberto, Mazza

Romel, Mackelprang

Rosanna, Salvia

Rose, Baker

Ruoniu, Wang

Samina, Yasmeen

Sandra, Taylor

Sandro Nuno, Ferreira De Serpa

Sandro, Serpa

Sara, Bonati

Sara, Delamont

Sara, Nunes

Sarah, Pickard

Sarah, Richard

Sarojni, Choy

Sayyed Ali, Samadi

Sean T., Lyons

Serdar, Kaya

Shelly, Brown-Jeffy

Shervin, Assari

Shing-Chung Jonathan, Yam

Sibelan, Forrester
Silvia, Moscatelli

Silvia, Staubli

Simon, Harding

Simon, Stjernholm

Sonali, Kudva

Soudeh, Ghaffari

Stacey, Balkan

Stacey, Greene

Stephen, Bazen

Stephen, Cook

Steven, Webber

Stuart, Fox

Sue, Vice

SunMan, Yoon

Supriya, Akerkar

Susanna Maria, Taraschi

Suzanne, Huot

Tahir, Abbas

Theo, Gavrielides

Thomas, Bienengräber

Tiffany A., Dykstra-DeVette

Timothy C., Baker

Tinios, Platon

Todd I., Herrenkohl

Tom, VanHeuvelen

Tone, Hellesund

Tonya, Sanders

Trevor, Herbert

Uma, Segal

Veronica, Barnsley

Vincent, Kaufmann

Vitor Sérgio, Ferreira

Weiqiang, Lin

William P., Baumgarth

Wouter, Zwysen

$\mathrm{Wu}$, Hui-Ching

Yalda, Hamidi

Yasmin, Jiwani

Yda J., Smith

Yi-Ping, Tseng

Youjung, Lee

YuChun, Yao

Yulia, Egorova

Yulin, Chen

(C) 2019 by the author. Licensee MDPI, Basel, Switzerland. This article is an open access article distributed under the terms and conditions of the Creative Commons Attribution (CC BY) license (http://creativecommons.org/licenses/by/4.0/). 